\title{
Patient engagement in pediatric concussion research
}

\author{
Nick Reed PhD MScOT, Kathy Leeder BA, Roger Zemek MD; for the PedCARE Research Team
}

Cite as: CMAJ 2018;190(Suppl 1):S28-S30. doi: 10.1503/cmaj.180450

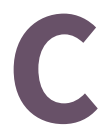

oncussion is a brain injury that affects hundreds of thousands of Canadian children and youth annually, along with their families. Visit rates for concussion have quadrupled since 2003, ${ }^{1}$ with nearly 30000 doctor visits for pediatric concussion per year in Ontario alone. ${ }^{2}$ Concussion can result in symptoms across four common domains: physical, cognitive, emotional and sleep. ${ }^{3}$ These symptoms can affect quality of life and result in short- and long-term functional deficits (e.g., in school, sports and social activities). ${ }^{4}$

A prospective, pan-Canadian study involving more than 3000 children and youth with acute concussion reported that about one-third continued to experience persistent symptoms one month after the injury. ${ }^{5}$ Despite strong evidence showing the benefits of early exercise for physical and mental health in individuals with moderate-to-severe brain injuries and stroke, ${ }^{6}$ traditional approaches to concussion management emphasize conservative cognitive and physical rest until postconcussion symptoms have resolved, ${ }^{7}$ which may hinder recovery.

The Pediatric Concussion Assessment of Rest and Exertion (PedCARE) study (ClinicalTrials.gov NCT02893969) is an ongoing randomized controlled trial that examines whether early reintroduction of noncontact and low-risk (e.g., limited chance of sustaining a concussion) physical activity, such as walking, light jogging or stationary cycling, beginning 72 hours postinjury, reduces postconcussion symptoms at two weeks postinjury compared with the traditional "rest until asymptomatic" protocol. ${ }^{8}$ The PedCARE study aims to provide health care professionals with evidence to guide the ideal timing for reintroduction of physical activity postconcussion and, more importantly, help children and youth successfully return to the things they need, want and love to do.

Guided by the four core principles of quality engagement reported by Abelson and colleagues ${ }^{9}$ (integrity of design and process, influence and impact, participatory culture, and collaboration and common purpose), the PedCARE group has attempted to engage patients and their families purposefully and meaningfully at all stages of the project. The remainder of this commentary presents a selection of PedCARE's patient-engagement activities and experiences.

\section{KEY POINTS}

- Traditional recommendations for concussion management emphasize cognitive and physical rest, which may hinder recovery.

- New approaches to postconcussion treatment must be explored; the reintroduction of noncontact and low-risk physical activity in the acute stages after injury may be beneficial.

- It is imperative to have the input of patients and families to guide concussion research and related implementation for improved clinical care.

- The use of qualitative interviews with patients and end-users to inform research study design, consultation with existing and established patient-engagement platforms, and question development for feedback from research participants at the time of research data collection have resulted in a more relevant clinical research study (the Pediatric Concussion Assessment of Rest and Exertion study) with outcomes that truly matter to children, youth and their families.

In the summer and fall of 2015, proactive qualitative interviews (via telephone) were completed with 50 children and youth, parents and caregivers, teachers and sport coaches across three large Ontario cities (Ottawa, Toronto and Hamilton). These one-on-one 60-minute interviews helped to inform key patientengagement priorities relevant to our planned research (PedCARE): opinions and attitudes regarding the current standard of care (interest in activity over rest after concussion); thoughts on our proposed study intervention (interest in early reintroduction of physical activity); opinions regarding our study design; and selection and identification of research outcome priorities from the patient and the family perspective.

Our research team consulted existing patient-engagement platforms to support the development of our data-collection processes in advance of the research project. The Research Family Engagement Committee at the Holland Bloorview Kids Rehabilitation Hospital comprises family members of current and past clients. ${ }^{10}$ This committee partners with researchers to plan, review and advise on 


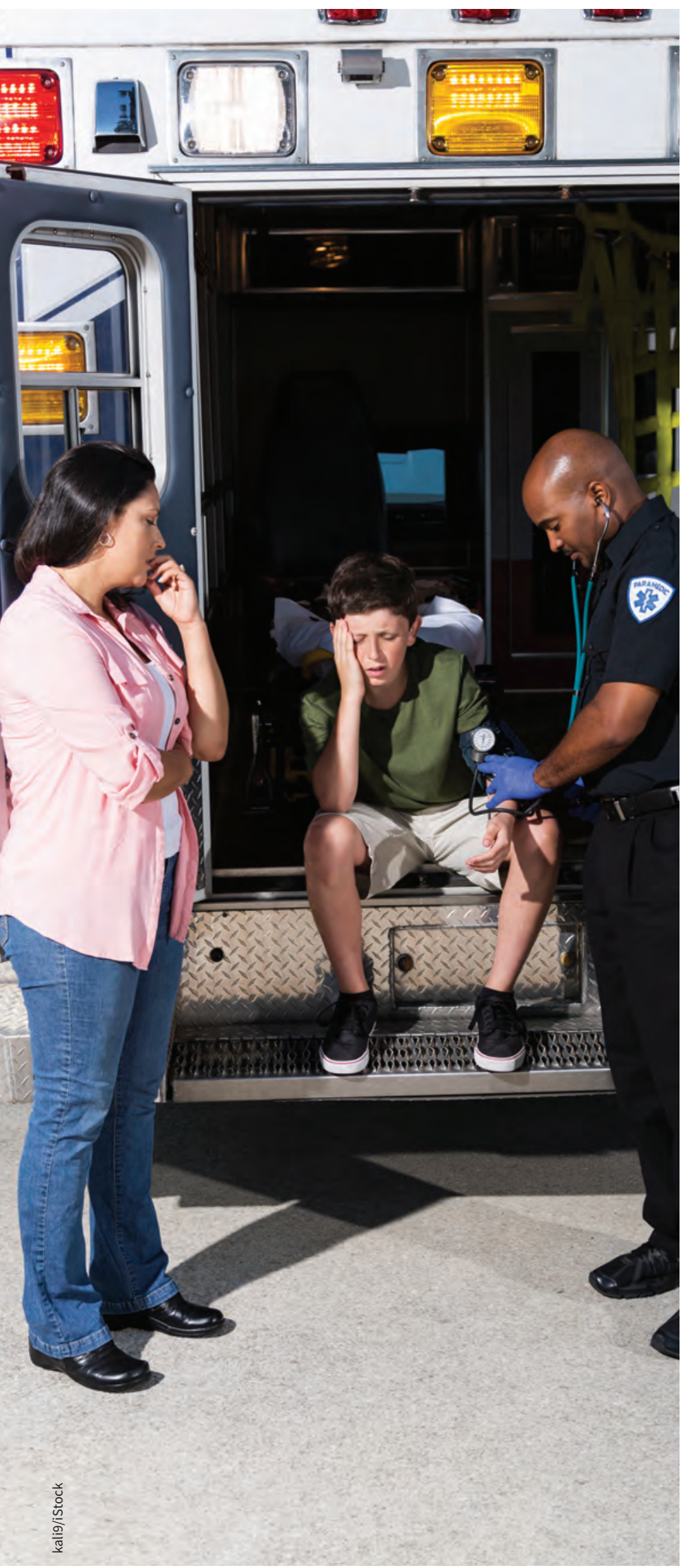

research studies. ${ }^{10}$ The consultation resulted in multiple committee members reviewing the content and wording of questions to be used during the data-collection phase of our research study (e.g., how to word demographic questions and responses specific to child and youth participants identifying sex and gender). This feedback was invaluable and directly informed the data-collection process used within the study. This collaboration also resulted in identifying our PedCARE patient partner (commentary coauthor K.L.), who will support the remaining stages of the project.

Another element of patient engagement within the PedCARE study is the use of feedback questions for research participants. In addition to collecting data to inform the study's research objectives, we ask all participants (and families) to provide their perspectives on the following questions: What did you like about the study? What were the challenges to completing the study? When the study is completed, how do you think we should share the results with others (kids, parents, health professionals, etc.)? Would you be willing to be contacted to provide feedback on the ways we plan to share the results of the study? Are you interested in being part of the study advisory committee?

We received specific feedback pertaining to the elastic belt that is used to hold the accelerometer used in our study. Based on this feedback, we obtained different belt sizes to meet the needs of participants. We received valuable suggestions for recipients of eventual results (e.g., youth, schools, sports teams, health professionals and hospitals), how best to share results with others and advance the eventual health system implementation and uptake (e.g., Facebook, email and inperson information sessions), and how to improve future studies or related clinical protocols (e.g., shorter questionnaires and decrease the need for daily tasks and reporting). This approach has also provided us with a roster of children, youth and family members who are interested in contributing to the project on our developing advisory committee.

A challenge experienced during our patient-engagement experiences to date was the need for considerable time and resources to conduct our qualitative interviews, analyze our data on feedback questions for research participants, and coordinate patient-engagement activities. The value of these approaches was substantial; however, the need for ample time and resources (people and financial) should be considered and accounted for well in advance during the planning of future research studies and patient-engagement initiatives.

Engaging patients in the project development and delivery of PedCARE has provided essential and invaluable insight, and has resulted in more relevant research. In addition to our published protocol, ${ }^{8}$ our research team looks forward to sharing the results of this ongoing clinical trial more broadly (i.e., scientific publications, presentations and patient-facing resources), once available, to affect patient care and outcomes directly. We will continue to engage patients and families as our project progresses from the data collection phase toward data analysis and the dissemination of findings. PedCARE aims to create a legacy for improved pediatric concussion care to best meet the growing needs of children and families in Ontario and 
beyond - it is our strong belief that achieving this aim is possible only with the perspectives and meaningful partnership of research participants and the children and families who will benefit from this research the most.

\section{References}

1. Zemek RL, Grool AM, Rodriguez Dugue D, et al. Annual and seasonal trends in ambulatory visits for pediatric concussion in Ontario between 2003 and 2013. J Pediatr 2017;181:222-228.e2.

2. Fridman L, Scolnik M, Macpherson A, et al. Annual trends in follow-up visits for pediatric concussion in emergency departments and physicians' offices. J Pediatr 2018;192:184-8.

3. Davis GA, Anderson V, Babl FE, et al. What is the difference in concussion management in children as compared to adults? A systematic review. Br J Sports Med 2017;51:949-57.

4. Novak Z, Aglipay M, Barrowman N, et al. Association of persistent postconcussion symptoms with pediatric quality of life. JAMA Pediatr 2016;170:e162900.
5. Zemek R, Barrowman N, Freedman SB, et al. Clinical risk score for persistent postconcussion symptoms among children with acute concussion in the ED. JAMA 2016;315:1014-25.

6. Pollock A, Baer G, Campbell P, et al. Physical rehabilitation approaches for the recovery of function and mobility following stroke. Cochrane Database Syst Rev 2014;(4):CD001920.

7. McCrory P, Meeuwisse WH, Aubry M, et al. Consensus statement on concussion in sport: the 4th International Conference on Concussion in Sport held in Zurich, November 2012. Br J Sports Med 2013;47:250-8.

8. Ledoux AA, Barrowman NJ, Boutis $\mathrm{K}$, et al. Multicentre, randomized clinical trial of paediatric concussion assessment of rest and exertion (PedCARE): a study to determine when to resume physical activities following concussion in children. Br J Sports Med 2017 Jul 12. pii: bjsports-2017-097981.

9. Abelson J, Li K, Wilson G, et al. Supporting quality public and patient engagement in health system organizations: development and usability testing of the Public and Patient Engagement Evaluation Tool. Health Expect 2016;19:817-27.

10. Research Family Engagement Committee. Client and family resources. Toronto: Holland Bloorview Kids Rehabilitation Hospital. Available: https:// hollandbloorview.ca/clientfamilyresources/clientfamilycentredcare/research familyengagementcommittee (accessed 2018 Apr. 2).
More information on this project is available at www.ossu.ca/ IMPACTAwards.

Competing interests: Roger Zemek is supported by the University of Ottawa's Brain and Mind Research Institute with a Clinical Research Chair in Pediatric Concussion. Nick Reed is supported by the Holland Family Chair in Acquired Brain Injury, a hospital-university named chair with Holland Bloorview Kids Rehabilitation Hospital and the University of Toronto. No other competing interests were declared.

This article was solicited and has been peer reviewed.

Affiliations: Holland Bloorview Kids Rehabilitation Hospital (Reed, Leeder), Department of Occupational Science and Occupational Therapy, University of Toronto (Reed), Toronto, Ont.; Children's Hospital of Eastern Ontario Research Institute (Zemek), Departments of Pediatrics and Emergency Medicine, University of Ottawa (Zemek), Ottawa, Ont.
Contributors: Nick Reed drafted the commentary, which Kathy Leeder and Roger Zemek revised. All of the authors gave final approval of the version to be published and agreed to be accountable for all aspects of the work.

Funding: This research and related patient-engagement activities were supported by OSSU (the Ontario SPOR [Strategy for Patient-Oriented Research] SUPPORT [Support for People and Patient-Oriented Research and Trials] Unit) (Ontario Child Health Support Unit IMPACT-Child Health Award). Additionally, the pre-project qualitative interviews used to inform patient-engagement priorities relevant to this research were supported by the Ontario Neurotrauma Foundation.

Correspondence to: Nick Reed, nreed@hollandbloorview.ca 